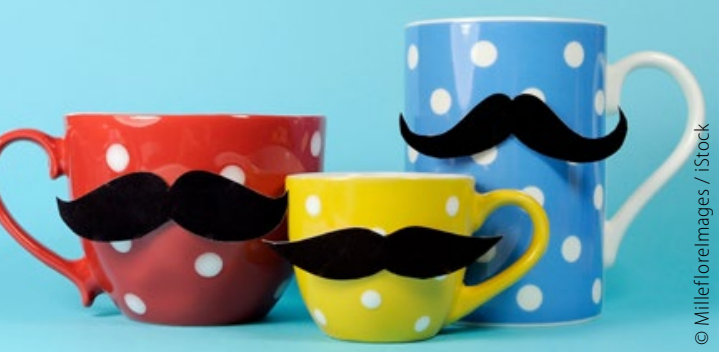

Zwei Tassen Kaffee für die Potenz?

Kaffeetrinker leiden seltener an einer erektilen Dysfunktion. Das gilt vor allem bei einem Konsum von zwei bis drei Tassen pro Tag. Die Analyse der NHANES-Kohorte anhand von Daten von 3724 Männern stützt den inversen Zusammenhang. Die Assoziation ist allerdings abhängig von Komorbiditäten: Bei Übergewicht und Bluthochdruck zeigte sich der Zusammenhang zwischen Koffeinzufuhr und Erektionsfähigkeit. Bei Männern mit normalem Gewicht, normalem Blutdruck und/oder mit Diabetes bestand kein signifikanter Zusammenhang.

Lopez DS et al. PLoS ONE 10(4): e0123547

\section{„Pille danach" boomt}

Nach der Entlassung der Notfallkontrazeptiva aus der Verschreibungspflicht steigt die Nachfrage: Der Verbrauch ist stark angestiegen, berichten die Marktforschungsinstitute IMS Health und Insight Health. Lag der Monatsabsatz im vergangenen Jahr in etwa konstant bei 37.000 bis 44.000 abgegebenen Packungen, damals noch von Ärzten rezeptiert, stieg die Menge schon im März binnen zwei Wochen auf über 50.000 Packungen. Im April wurden 55.000 verkauft, so Insight Health.

www.aerztezeitung.de

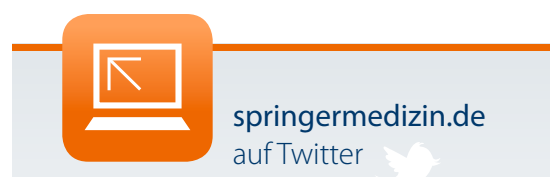

Auch im Frühsommer heißt es

"Lauschen" Sie unserem

Zwitschern und werden Sie zum

"Follower"

- twitter.com/springermedizin
Junge Krebspatientinnen mit positiver Familienanmnese haben öfter ungünstige Tumoreigenschaften. Ihr Rezidivrisiko ist mit den heute üblichen Therapien aber nicht erhöht.

Nach Ergebnissen der POSH (Prognostic Outcomes in Sporadic vs. Hereditary breast cancer)-Studie ist die Sorge junger Frauen unbegründet. „Die Familiengeschichte ist mit den heutigen Therapien kein unabhängiger Risikofaktor für ein Brustkrebsrezidiv“, betonen die Studienautoren um Bryony K. Eccles von der Universität Southampton. Von 2850 Teilnehmerinnen waren $34,1 \%$ familiär vorbelastet (FH+). Die Vorbelastung lässt Frauen bei Auffälligkeiten früher einen Arzt aufsuchen, sie weisen aber auch eine höheres
Grading bei den Tumoren auf: Tumoren wurden erwartungsgemäß häufiger im Rahmen von Kontrollen entdeckt (3,0 FH+ vs. 0,1\% FH-). Sie wurden häufiger als Grad 3 klassifiziert $(63,3$ vs. $58,9 \%)$ und waren seltener HER-2-positiv (24,7 vs. $28,8 \%$ ).

\section{Nicht mehr Fernmetastasen}

Die chirurgische Therapie bestand in der $\mathrm{FH}+-$ Gruppe häufiger in einer Mastektomie $(54,4$ vs. $48,5 \%)$. Bei der Chemotherapie gab es keine Gruppenunterschiede. Nach fünf Jahren waren geschätzt 74,9\% der Patientinnen ohne und $77,4 \%$ mit familiärer Vorbelastung frei von Fernmetastasen. Die Differenz war statistisch jedoch nicht signifikant. (wg)

Eccles BK et al. Br J Surgery 2015 (online first)

\title{
Erhöhtes Risiko beobachtet
}

\section{Psoriasis: Schwere Infekte durch Biologika?}

Aus der PSOLAR-Studie geht hervor, dass während der Psoriasistherapie die Wahrscheinlichkeit für schwere Infektionen bei Therapie mit den derzeit gebräuchlichen Biologika unterschiedlich hoch ist, verglichen mit Nicht-Biologika.

In der Beobachtungsstudie PSOLAR (Psoriasis Longitudinal Assessment and Registry) wird die Sicherheit und Wirksamkeit der Psoriasistherapie untersucht, wobei das gesamte derzeit verfügbare Arzneispektrum berücksichtigt wird. Bei den Biologika handelt es sich um Ustekinumab, Infliximab, Adalimumab und Etanercept. Unter den Medikamenten, die nicht gentechnisch hergestellt sind, befinden sich Kortikosteroide, Ciclosporin und Methotrexat. In dem achtjährigen Zeitraum wur- den bei insgesamt 11.466 Patienten insgesamt 323 schwere Infektionen dokumentiert. Bezogen auf die Gesamtpopulation der Studienteilnehmer betrug die Rate schwerer Infektionen unter Ustekinumab 0,83, Etanercept 1,47, Adalimumab 1,97 und Infliximab 2,49 pro 100 Patientenjahre. In der Gruppe ohne Biologika und ohne Methotrexat lag der Wert bei 1,05 und in der Gruppe ohne Biologika, aber mit Methotrexat bei 1,28 pro 100 Patientenjahre. Die häufigsten Infektionen waren Pneumonien und Zellulitis. Wie erwartet, seien Alter, Diabetes, Infektionen in der Anamnese sowie Rauchen signifikant mit dem Auftreten schwerer Infektionen assoziiert gewesen, so die Forscher.

(wg)

Kalb RE et al. JAMA Dermatol 2015 (online first), doi: 10.1001/jamadermatol.2015.0718 